\title{
Survival of SARS-COV-2 under liquid medium, dry filter paper and acidic conditions
}

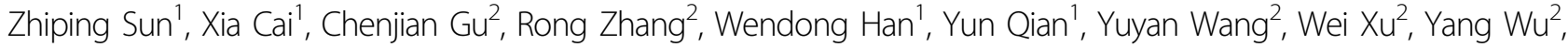 \\ Xunjia Cheng' ${ }^{2}$ Zhenghong Yuan'2, Youhua Xie ${ }^{2}$ and $\mathrm{Di}^{\mathrm{Qu}} \mathrm{u}^{1,2}$
}

Dear Editor,

The pneumonia caused by a novel coronavirus was first reported in December 2019 in Wuhan of China, and since then has become a pandemic ${ }^{1,2}$. International Committee on Taxonomy of Viruses (ICTV) named the virus as severe acute respiratory syndrome coronavirus 2 $(\mathrm{SARS}-\mathrm{CoV}-2)^{3}$. SARS-COV-2 is transmitted mainly through respiratory droplets and close contact ${ }^{4}$. The fast spread of the coronavirus disease (COVID-19) ${ }^{3}$ suggests that SARS-COV-2 is highly contagious. The virus remained viable in the medium for 7 days at $22^{\circ} \mathrm{C}$ and 1 day at $37^{\circ} \mathrm{C}^{5}$. On dry surfaces at room temperature (RT), the virus was reported viable for 1 day on the surface of cloth, for 4 days on stainless steel, and for 7 days on the outer layer of a surgical mask, whereas no infectious virus was recovered from the surfaces of printing and tissue papers after a 3 -h incubation ${ }^{5}$. Here, we first investigated the infectivity of SARS-COV-2 using a plaque-purified strain nCoV-SH01 isolated from a patient in Shanghai (GenBank MT121215) ${ }^{6}$, studied subsequently its stability in liquid medium, on dry filter paper, and under acidic condition (pH2.2) at RT. It would provide guidance on application appropriate measures to control the spread of SARS-COV-2 and improve laboratory biosafety management.

First the virus stock of nCoV-SH01 was quantified on Vero-E6 cells by plaque forming assay (plaque forming unit) and $\mathrm{TCID}_{50}$ assay (tissue culture infection dose), as

\footnotetext{
Correspondence: Zhenghong Yuan (zhyuan@shmu.edu.cn) or

Youhua Xie (yhxie@fudan.edu.cn) or Di Qu (dqu@shmu.edu.cn)

'BSL-3 Laboratory of Fudan University, School of Basic Medical Sciences, Shanghai Medical College, Fudan University, Shanghai, China

${ }^{2}$ Key Laboratory of Medical Molecular Virology (MOE/NHC/CAMS), Department of Medical Microbiology and Parasitology, School of Basic Medical Sciences,

Shanghai Medical College, Fudan University, Shanghai, China

These authors contributed equally: Zhiping Sun, Xia Cai, Chenjian Gu,

Rong Zhang
}

$6 \times 10^{5} \mathrm{PFU} / \mathrm{mL}$ and $2.8 \times 10^{6} \mathrm{TCID}_{50} / \mathrm{mL}$, respectively. Cytopathic effects (CPE) appeared at $24 \mathrm{~h}$ post inoculation (h.p.i.) with $100-2000 \mathrm{PFU}$ of the virus titer, at 48 h.p.i. with 5-50 PFU (Supplementary Fig. S1a), and at 72 h.p.i. with 1 PFU virus (Table 1a and Supplementary Fig. S1b). Based on that, we used $1.2 \times 10^{3}$ PFU $\left(3.75 \log _{10} \mathrm{TCID}_{50}\right)$ virus in subsequent experiments.

SARS-COV-2 can be shed into wet or dry surrounding by droplets or aerosol ${ }^{4}$. How stable is the virus in different environment? We first determined viral stability in liquid medium. $1.2 \times 10^{3}$ PFU (3.75 $\log _{10}$ TCID $_{50}$ ) virus in DMEM was added into each well kept in a wet box at RT. After set for $1,2,3,4,5,6$, or 7 days, respectively, $100 \mu \mathrm{L}$ of the virus solution was transferred from each sample onto Vero-E6 monolayer. CPE were checked daily till day 5 . We found that when the virus had been kept in the medium at RT for 1 day, CPE appeared at 24 h.p.i., which was like the untreated virus control. When the virus had been kept for 2 or 3 days, CPE emerged at 48 h.p.i. (Table 1b). By day 3, the virus titer decreased 2 Logs (from 3.75 to $1.35 \log _{10} \mathrm{~T}$ $\mathrm{CID}_{50}$ ). When the virus had been left in the medium for more than 4 days, no CPE was observed. The loss of infectivity was confirmed by $\mathrm{TCID}_{50}$ assay, immune florescence staining with the antiserum against viral $\mathrm{N}$ protein (Supplementary Fig. S2a) and qRT-PCR (Ct value over the cutoff $>38$, Supplementary Table S1). We then investigated viral stability on dry filter paper at RT. $1.2 \times 10^{3}$ PFU ( 3.75 $\log _{10} \mathrm{TCID}_{50}$ ) virus in $5 \mu \mathrm{L}$ DMEM was added onto sterilized filter paper in plates. After completely dried, the plates were put into a dry box at RT. After set for $1,2,3,4,5,6$, or 7 days, the virus on the filter paper was eluted with DMEM, respectively. The eluted virus titer was $3.42 \log _{10} \mathrm{TCID}_{50}$ after the virus remained on the paper air dried for $1 \mathrm{~h}$ (recovery efficiency was $10^{3.42} / 10^{3.75}=10^{-0.33}=46.77 \%$ ) and CPE appeared on day 2 post inoculation. When the virus had been kept on dried filter paper for 1 or 2 days at 


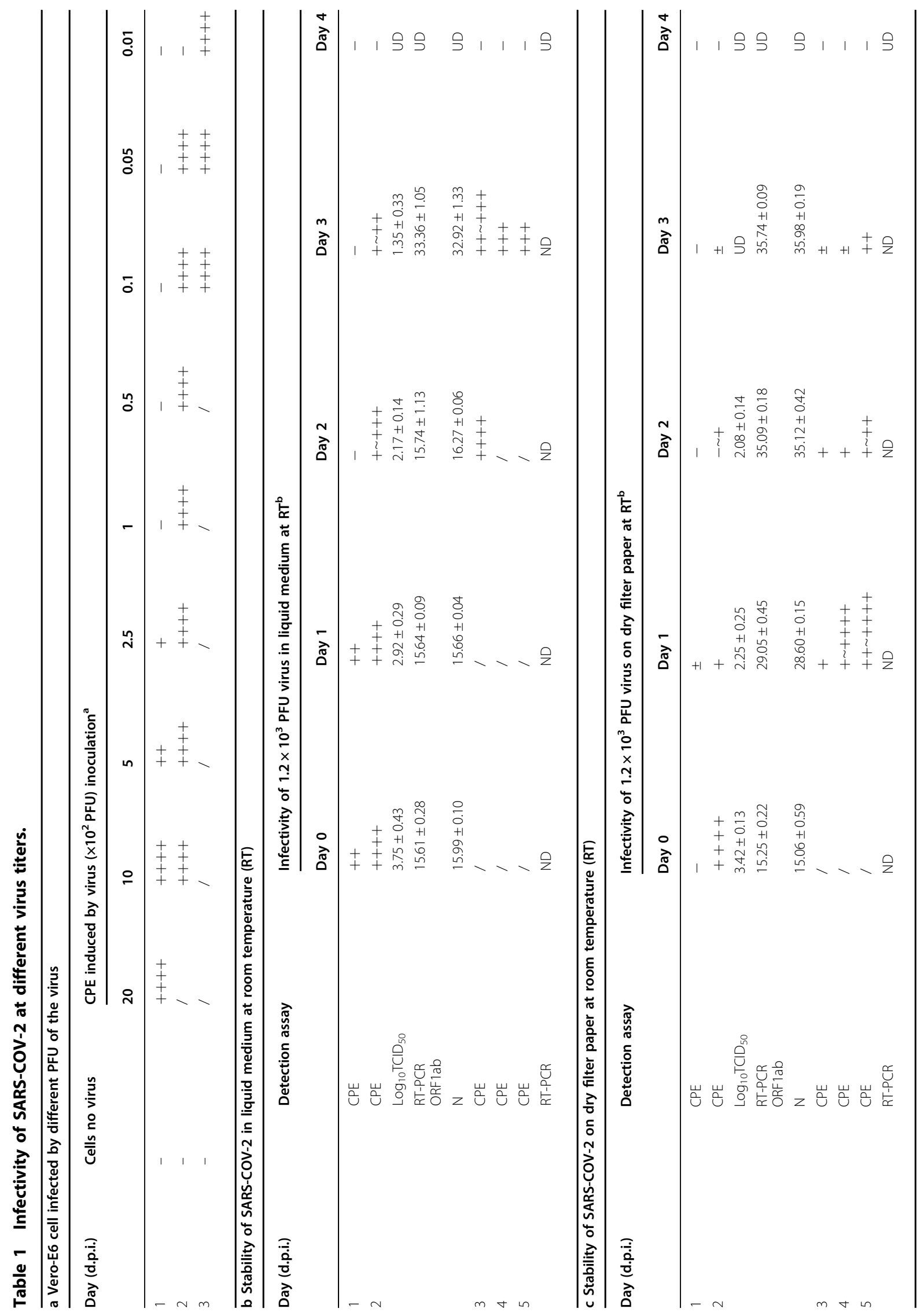




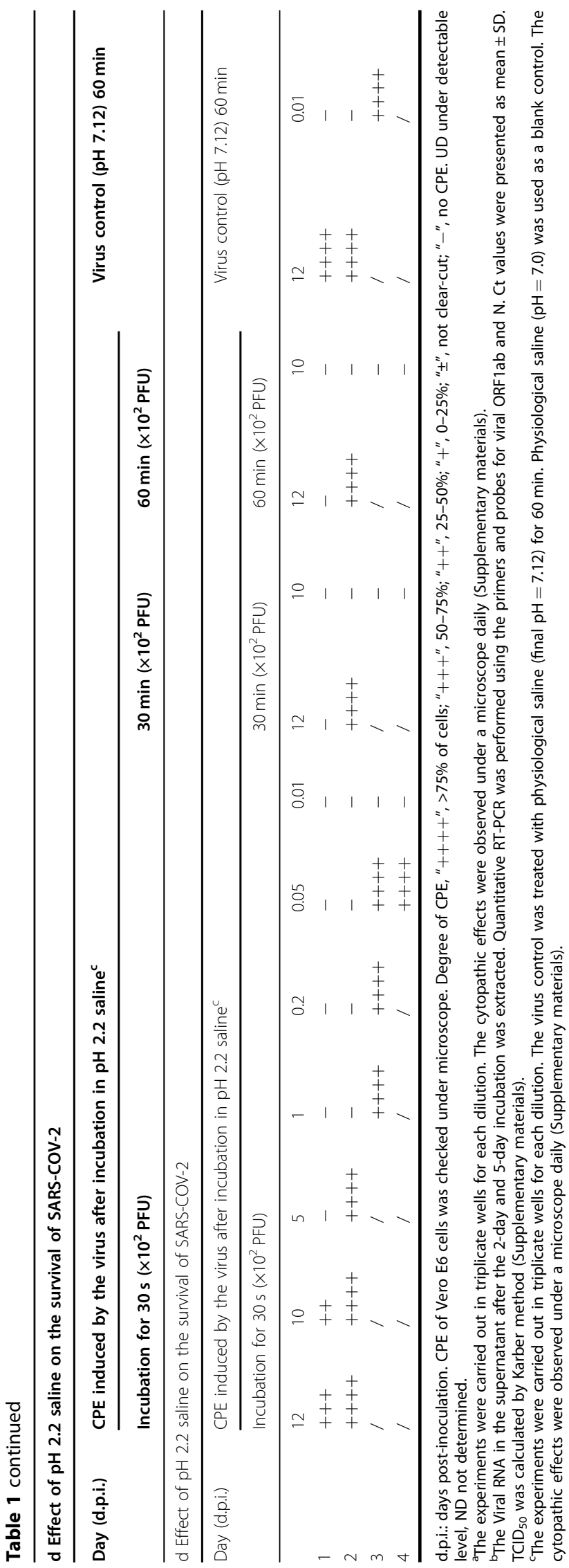

RT, CPE appeared on day 4 or 5 post inoculation, respectively (Table 1c), and the virus titer dropped to 2.17 $\log _{10} \mathrm{TCID}_{50}$ with the 2-day incubation. For the 3-day incubation at RT, CPE appeared at day 5 post inoculation but the virus titer could not be determined by $\mathrm{TCID}_{50}$ assay, and for the 4-day incubation, no CPE was observed. The loss of infectivity was also confirmed by immune florescence staining with the anti-N serum (Supplementary Fig. S2b) and qRT-PCR. Our results show that COVID-19 virus can survive for 3 days in liquid medium or on dry filter paper. For the 3-day incubation in liquid medium at RT, viable virus left only $1.35 \log _{10} \mathrm{TCID}_{50}$ (initial titer was 3.75). For the 3-day incubation on dry filter paper at RT, although CPE was observed, the survived virus could not be quantified by $\mathrm{TCID}_{50}$ assay. The loss of virus viability in prolonged incubation ( $>4$ days) was confirmed by $\mathrm{N}$ protein immunofluorescence staining, qRT-PCR, and further verified by blind passage of the supernatant for three generations. In Alex's study, the virus remained viable in the medium for 7 days at $22^{\circ} \mathrm{C}^{5}$. The reason for the longer survival time in their report might be the higher viral titer they used $\left(\sim 6.7 \log _{10} \mathrm{~T}\right.$ $\mathrm{CID}_{50}$ versus $3.75 \log _{10} \mathrm{TCID}_{50}$ in this study). Regardless, our results show that SARS-COV-2 is highly infectious and relatively stable in the environment, which underscores the importance of environmental disinfection and hand hygiene.

Since some coronaviruses can cause infectious diseases of digestive tract via gastrointestinal transmission, such as mouse hepatitis virus (MHV), porcine epidemic diarrhea virus (PEDV), and feline enteric coronavirus $(\mathrm{FECV})^{7-9}$. Bioinformatics analysis of single-cell transcriptomes revealed that ACE2 was expressed in esophagus squamous epithelium cells and enterocytes of ileum and $\operatorname{colon}^{10}$, hence SARS-COV-2 might be potentially transmitted via the fecal-oral route ${ }^{11,12}$. We speculate that SARS-COV-2 would have to survive the gastric acidic environment if the virus is indeed transmitted via the fecal-oral route. Consequently, we determined the survival of SARS-COV-2 under acidic condition in vitro. Various amount of the virus $(1.2 \times$ $10^{3}, \quad 1.0 \times 10^{3}, \quad 5 \times 10^{2}, \quad 1 \times 10^{2}, \quad 0.2 \times 10^{2}, \quad 0.05 \times 10^{2}$, $\left.0.01 \times 10^{2} \mathrm{PFU}\right)$ was treated with acidic physiological saline $(\mathrm{pH} 2.2)$ at $\mathrm{RT}$ for $30 \mathrm{~s}, 30 \mathrm{~min}$ or $60 \mathrm{~min}$, respectively. After treatment, each viral sample was adjusted to $\mathrm{pH} 7.28$ and added onto Vero-E6 monolayer. As shown in Table 1d, after a 30-s incubation in $\mathrm{pH}$ 2.2 saline, significant $\mathrm{CPE}$ appeared at 48 h.p.i. with $1.2 \times 10^{3}, 1.0 \times 10^{3}, 5 \times 10^{2}$, or $1 \times 10^{2} \mathrm{PFU}$ of the virus. CPE appeared at 72 or 96 h.p.i. with lower virus titers $\left(1 \times 10^{2}, 0.2 \times 10^{2}\right.$, or $\left.0.05 \times 10^{2} \mathrm{PFU}\right)$. No CPE was seen with $0.01 \times 10^{2} \mathrm{PFU}$ virus while $\mathrm{CPE}$ from the same amount as the virus control $\left(0.01 \times 10^{2} \mathrm{PFU}\right)$ was readily observed at 72 h.p.i. After the $30-\mathrm{min}$ or 60 -min 
incubation in $\mathrm{pH} 2.2$ saline, no CPE was observed with the virus titers equal and below $1.0 \times 10^{3} \mathrm{PFU}$, whereas $\mathrm{CPE}$ appeared with $1.2 \times 10^{3} \mathrm{PFU}$ virus although the survived virus could not be determined by TCID $_{50}$ assay (Table $1 \mathrm{~d}$ and Supplementary Fig. S3) and confirmed by immune florescence staining (Supplementary Fig. S4). Transmission of SARS-COV-2 through the fecal-oral route is currently uncertain. Although SARS-COV-2 RNA has been detected in patients' stool ${ }^{11,13,14}$, infectious virus was not readily isolated from stool. In the present study, when $1.2 \times 10^{3} \mathrm{PFU}$ virus was treated with the acidic saline of $\mathrm{pH} 2.2$ for 30 or $60 \mathrm{~min}$, virus survival could be observed as manifested by CPE but failed to be quantified, whereas no apparent virus survival was detected with lower virus titers $\left(<1.0 \times 10^{3} \mathrm{PFU}\right)$ treated under the same acidic condition. The results suggest that SARS-COV-2 at a certain high titer might survive the acidic environment of the stomach for a certain period. Although it is unclear whether the virus can replicate in the intestine, the survived virus in the gastrointestinal tract may be excreted in faeces, which would indicate the importance of stool disinfection. Whether the virus can be transmitted through the fecal-oral route needs further study.

In conclusion, our findings show that SARS-COV-2 can survive for 3 days in liquid medium or on dry filter paper, and the virus at a high titer can survive under acidic condition that mimics the gastric environment. Our study would provide guidance on application of appropriate measures to control the spread of SARS-COV-2 and improve laboratory biosafety.

\section{Acknowledgements}

This work was supported by NSTMP (2018ZX10734401, 2018ZX10301208, 2019ZX09721001), Development Programs for COVID-19 of Shanghai Science and Technology Commission (Grant No. 20431900401) and Project of Novel Coronavirus of Fudan University.

\section{Author contributions}

Z.S., X.C., C.G., and R.Z. performed the viral experiment in BSL-3 lab, analyzed the data and participated in writing the paper. W.H., Y.Q., Yy.W., W.X., Y.W., and X.C. participated in experiments in BSL-3 lab. D.Q., Y.X., and Z.Y. designed the experiments, planned the approach, wrote and edited the paper.
Conflict of interest

The authors declare that they have no conflict of interest.

\section{Publisher's note}

Springer Nature remains neutral with regard to jurisdictional claims in published maps and institutional affiliations.

Supplementary Information accompanies the paper at (https://doi.org/ 10.1038/s41421-020-00191-9).

Received: 12 April 2020 Accepted: 1 July 2020

Published online: 14 August 2020

\section{References}

1. Wang, C., Horby, P. W., Hayden, F. G. \& Gao, G. F. A novel coronavirus outbreak of global health concern. Lancet 395, 470-473 (2020).

2. The 2019-nCoV Outbreak Joint Field Epidemiology Investigation Team, Q. L. Notes from the field: an outbreak of NCIP (2019-nCoV) Infection in China-Wuhan, Hubei Province 2019-2020. China CDC Wkly. 2, 79-80 (2020).

3. World Health Organization. Naming the coronavirus disease (COVID-19) and the virus that causes it. https://www.who.int/emergencies/diseases/novelcoronavirus-2019/technical-guidance/naming-the-coronavirus-disease-(covid2019)-and-the-virus-that-causes-it (2020).

4. Guo, Y. R. et al. The origin, transmission and clinical therapies on coronavirus disease 2019 (COVID-19) outbreak-an update on the status. Mil. Med. Res. 7, 11 (2020).

5. Chin, A. W. H. et al. Stability of SARS-CoV-2 in different environmental conditions. Lancet Microbe 1, e10 (2020).

6. Zhang Rong, Y. Z. et al. Isolation of a 2019 novel coronavirus strain from a coronavirus disease 19 patient in Shanghai. J. Microbes Infect. 15, 15-20 (2020).

7. Compton, S. R. et al. Pathogenesis of enterotropic mouse hepatitis virus in immunocompetent and immunodeficient mice. Comp. Med. 54, 681-689 (2004).

8. Tekes, G. \& Thiel, H. J. Feline coronaviruses: pathogenesis of feline infectious peritonitis. Adv. Virus Res. 96, 193-218 (2016).

9. Li, W. van Kuppeveld, F. J. M., He, Q., Rottier, P. J. M. \& Bosch, B. J. Cellular entry of the porcine epidemic diarrhea virus. Virus Res. 226, 117-127 (2016)

10. $\mathrm{Xu}, \mathrm{H}$. et al. High expression of ACE2 receptor of 2019-nCoV on the epithelial cells of oral mucosa. Int. J. Oral. Sci. 12, 8 (2020).

11. Zhang, T. et al. Detectable SARS-CoV-2 viral RNA in feces of three children during recovery period of COVID-19 pneumonia. J. Med. Virol. 92, 909-914 (2020).

12. Gu, J., Han, B. \& Wang, J. COVID-19: gastrointestinal manifestations and potential fecal-oral transmission. Gastroenterology 158, 1518-1519 (2020).

13. Pan, Y., Zhang, D., Yang, P., Poon, L. L. M. \& Wang, Q. Viral load of SARS-CoV-2 in clinical samples. Lancet Infect. Dis. 20, 411-412 (2020).

14. Wolfel, R. et al. Virological assessment of hospitalized patients with COVID2019. Nature 581, 465-469 (2020). 